Bundesgesundheitsbl -

Gesundheitsforsch - Gesundheitsschutz

2001 • 44:960-965 @ Springer-Verlag 2001

Originalien und Übersichtsbeiträge

S.N.Willich $\cdot$ A.M.Sharma ${ }^{2} \cdot$ E. O. Krasemann ${ }^{3} \cdot$ W. Meyer-Sabellek ${ }^{4} \cdot$ W. Thefeld $^{5}$

${ }^{1}$ Institut für Sozialmedizin und Epidemiologie, Klinikum Charité der Humboldt-Universität, Berlin

${ }^{2}$ Franz-Volhard-Klinik, Klinikum Charité der Humboldt-Universität, Berlin

${ }^{3}$ Arbeitsgemeinschaft Herz-Kreislauf, Hamburg

${ }^{4}$ AstraZeneca GmbH, Wedel

${ }^{5}$ Robert Koch-Institut, Berlin

\title{
Epidemiologische, genetische und therapeutische Aspekte der Adipositas
}

"Let me have men around me that are fat..." (Lasst Männer um mich sein, die dick sind).

Bei Shakespeare deutet Julius Caesar Index $\geq 30 \mathrm{~kg} / \mathrm{m}^{2}$ ) liegt in Deutschland für Männer bei $19,9 \%$ und für Frauen bei $21,6 \%$, im Osten jeweils höher als im Westen. Medizinische Risiken bei Übergewicht sind vor allem die erhöhte Inzidenz von Risikofaktoren und Manifestationen der Herz-KreislaufErkrankungen. Epidemiologische Untersuchungen zeigen zudem eine kontinuierliche Zunahme der Gesamtmortalität bereits ab Body Mass Index 25. In multivariaten Analysen erwies sich Adipositas unter Berücksichtigung von Hyperlipoproteinämie, Hypertonie und Diabetes als ein unabhängiger signifikanter Risikofaktor für koronare Herzkrankheit, Herzinsuffizienz, Schlaganfall und plötzlichen Herztod. Genetische Aspekte spielen bei der Entwicklung von Adipositas eine wichtige Rolle. Die Ergebnisse bisheriger Interventionsstudien sind weitgehend enttäuschend. Nach wie vor gilt:"Most obese persons will not stay in treatment, most will not lose weight, and of those who lose weight, most will regain it". („Die meisten adipösen Personen bleiben nicht in Behandlung, die meisten verlieren kein Gewicht und von denen, die Gewicht verlieren, nehmen die meisten wieder zu“.) Die Akzeptanz von Adipositas als ernst zu nehmende Erkrankung wird zur Entwicklung wirksamer und dauerhafter Therapie- und Präventionsstrategien beitragen.

\section{Schlüsselwörter}

Adipositas · Body Mass Index · Herz-Kreislauf-Erkrankungen · Risikofaktor im 1. Akt des gleichnamigen Dramas mit dem Wunsch nach beleibteren Männern in seiner Umgebung die Verbindung zwischen Körpergewicht und psychischer Disposition an, nämlich die seines Erachtens eher gefährlichen Seiten von hageren Männern [1]. Damals standen die medizinischen Probleme von Adipositas noch nicht zur Debatte. Dann im 19. Jahrhundert beschrieb der Hamburger Arzt von Dusch jedoch ein Ungleichgewicht zwischen Kalorienzufuhr und -verbrauch als mutmaßliche Ursache der koronaren Herzkrankheit [2]:,Denn namentlich leiden oft wohlhabende und reiche Leute an Angina Pectoris, welche den Genüssen einer reichlichen und luxuriösen Tafel sich ergeben, ohne die nötige körperliche Bewegung zu haben." 1969 wurde in Deutschland die aktive Rehabilitationstherapie in speziellen Kliniken für Patienten mit Herz-Kreislauf-Erkrankungen eingeführt, um spezielle Risikofaktoren und krankmachende Lebensbedingungen bei Patienten mit atherosklerotisch bedingten Krankheiten positiv zu verändern. Wissenschaftlich erhärtet ist inzwischen, dass für bestimmte Krankheiten einschließlich koronare Herzkrankheit, Typ-2-Diabetes, Schlaganfall und arterielle Hypertonie die Adipositas ein ernster verschlimmernder Risikofaktor ist. Untersuchungen zeigten auch, dass Adipositas die Lebenserwartung insgesamt negativ beeinflusst.
Medizinische Kenntnisse allein reichen nicht aus, um Adipositas wirksam zu bekämpfen. Die vernachlässigte therapeutische Problematik der dauerhaften Verhaltensänderung ist bisher ungelöst. Es ist bisher nicht gelungen, ein entsprechendes Problembewusstsein in der Bevölkerung zu erzeugen. Hier haben die vielen Bücher, Broschüren und die Seiten füllenden Diätanleitungen der Zeitschriften nicht weiter geführt. Nur erheblicher Leidensdruck und eine engmaschige Nachbetreuung können beim Einzelnen etwas ausrichten. Dabei kommt erschwerend hinzu, dass unsere ärztlichen Bemühungen gegen einen allgemeinen gesellschaftlichen Trend gerichtet sind. Die Gesellschaft ist von Wertvorstellungen großer Gruppen geprägt, die Selbstverwirklichung, Freiheit, Freude und Glück als vorrangiges Ziel definieren. Selbstbeherrschung, kritische Selbsterkenntnis, Anstrengungen und Durchhaltevermögen werden als altmodische Tugenden eingeschätzt. Das medizinisch unverbindliche Wort „Wohlfühlgewicht" wird dem Arzt vom Patienten entgegengehalten. Es bedarf einer erheblichen interdisziplinären Anstrengung von Medizinern, Psychologen, Pädagogen, Soziologen und den Patienten selbst, vertreten in Selbsthilfegruppen, um das

Prof.Dr.Stefan N. Willich

Institut für Sozialmedizin und Epidemiologie, Klinikum Charité,

Humboldt-Universität zu Berlin, 10098 Berlin,

E-Mail: stefan.willich@charite.de 


\section{S.N.Willich · A.M.Sharma \\ E. O. Krasemann · W. Meyer-Sabellek W.Thefeld}

\section{Epidemiologic, Genetic, and Therapeutic Aspects of Obesity}

\begin{abstract}
In Germany, the prevalence of obesity (body mass index $\geq 30 \mathrm{~kg} / \mathrm{m}^{2}$ ) in men is $19,9 \%$ and in women $21,6 \%$. In both groups the prevalence is higher in the easter compared to the western part of Germany. Obesity leads to the risk factors and manifestation of cardiovascular diseases. In addition, epidemiologic investigations indicate a continous increase in mortality, already starting at a body mass index of 25 . In multivariate analyses, obesity proved as an independent significant risk factor for coronary heart disease, heart failure, stroke, and sudden cardiac death after adjustment for hyperlipidemia, high blood pressure and diabetes. Genetic aspects play an important role in the development of obesity. To a large extent, the results of previous intervention studies have been disappointing. Still it holds:"Most obese persons will not stay in treatment, most will not lose weight, and of those who lose weight, most will regain it".The acceptance of obesity as a serious disorder will contribute to the development of effective and long-lasting therapeutic and preventive strategies.
\end{abstract}

\section{Keywords}

Obesity - Body mass index . Cardiovascular diseases $\cdot$ Risk factor
Problem Adipositas anzugehen. In der sozial- und präventivmedizinischen Umsetzung sind dann schließlich die Gesundheitspolitiker in Verbindung mit der Sozialversicherung gefragt, unterstützt durch die Medien.

Die medizinischen Risiken bei bestehender Adipositas umfassen Cholelithiasis, respiratorische Dysfunktion, einige Krebsarten und Gichtarthrose [3]. Im Vordergrund steht besonders das Risiko hinsichtlich Gesamtmortalität und Herz-Kreislauf-Erkrankungen, auf das wir uns in diesem Übersichtsartikel beziehen.

\section{Prävalenz}

Adipositas wird unterschiedlich kategorisiert und definiert. Neben dem Gewicht, dem Verhältnis von Taillen- zu Hüftumfang und der Fettverteilung hat sich in epidemiologischen Studien das auf die Körperoberfläche bezogenen Gewicht ausgedrückt als Body Mass Index (BMI) in $\mathrm{kg} / \mathrm{m}^{2}$ als Maßeinheit bewährt. Ein BMI ab 25 gilt international als Grenzwert zur Adipositas, kategorisiert in geringgradige (BMI 25-29,9), mittelgradige $(30-39,9)$ und hochgradige Adipositas $(\geq 40)$. In den Leitlinien der Deutschen Adipositas-Gesellschaft sind gleiche BMI-Bereiche aufgeführt, eingeteilt in Grad I (Übergewicht), Grad II (Adipositas) und Grad III (extreme Adipositas) [4]. Je nach zugrunde liegender Definition wird die Prävalenz von Adipositas in der Erwachsenenbevölkerung zwischen 10 und $50 \%$ angegeben. Frauen sind in den meisten Studien häufiger betroffen als Männer.

Die Prävalenz von Adipositas ist seit den 5oer-Jahren angestiegen, parallel zumindest die ersten 20 Jahre - zu den Herz-Kreislauf-Erkrankungen [5].Standardisierte Daten wurden im weltweiten Vergleich seit Mitte der 8oer-Jahre im MONICA-Projekt erhoben [6]. Bei den Männern zwischen 35 und 64 Jahren lag der BMI im Median zwischen 23,4 (Peking) und 27,5 (Kaunas), bei Frauen zwischen 23,6 (Stanford und Glostrup) und 29,3 (Kaunas). In den deutschen Zentren lagen die entsprechenden Werte bei Männern zwischen 26 und 27 und bei Frauen zwischen 24 und 26.

Eine aktuelle Beurteilung der zeitlichen Trends von Adipositas in der Allgemeinbevölkerung Deutschlands ermöglichen die Daten des Bundes-Ge- sundheitssurveys 1998. Nimmt man einen BMI von $30 \mathrm{~kg} / \mathrm{m}^{2}$ als Grenzwert, haben 19,9\% aller Männer zwischen 25 und 69 Jahren Übergewicht (Abb. 1 oben), bei einem Grenzwert von 25 sind es fast $70 \%$. Insgesamt ist der Trend in den 9oer-Jahren weiter ansteigend. Es zeigt sich außerdem, dass die Werte in den östlichen Bundesländern etwas höher liegen als in den westlichen. Bei den Frauen ist die Prävalenz der Adipositas (BMI $\geq 30$ ) höher als bei den Männern. Einen BMI von $30 \mathrm{~kg} / \mathrm{m}^{2}$ als Grenzwert haben $21,6 \%$ aller Frauen (Abb. 1 unten), einen Grenzwert von 25 haben über $50 \%$. Im zeitlichen Verlauf scheint in den 9oer-Jahren die Adipositas bei Frauen kaum zuzunehmen. Auch bei den Frauen liegen die Werte in den östlichen höher als in den westlichen Bundesländern. Der unterschiedlichen Adipositasprävalenz entsprechend war $1998 \mathrm{der}$ Anteil an Personen ohne sportliche Betätigung in den östlichen Bundesländern höher als in den westlichen: Bei Männern 52\% versus $40 \%$ und bei Frauen $51 \%$ versus $46 \%$.

Der Zusammenhang zwischen sozialer Schicht und der Prävalenz von Adipositas (wie auch anderen kardiovaskulären Risikofaktoren) ist gut belegt. Je höher die soziale Schicht, umso geringer ist die Wahrscheinlichkeit des Vorliegens von Übergewicht, Hypertonus und Rauchen bei einer Person [7]. Bei Patienten mit bekannter koronaren Erkrankung liegt die Prävalenz von Adipositas mit einem BMI $\geq 30$ um $20 \%$ [8, 9]. Im Verlauf des ersten Jahres nach einer akuten Koronarerkrankung steigt die Prävalenz in dieser Risikopopulation sogar noch weiter an. Zusammenfassend nimmt die Prävalenz von Adipositas weltweit weiterhin zu, selbst bei Patienten mit bekanntem hohen koronaren Risiko.

\section{Body Mass Index als kardiovaskulärer Risikofaktor}

Die Zunahme des BMI erhöht das Risiko für kardiovaskuläre Erkrankungen im gleichen Maße wie der Anstieg des Cholesterinwertes und des diastolischen Blutdrucks [10], die entsprechenden Kurven verlaufen parallel (Abb. 2). Eine Gewichtserhöhung um $10 \mathrm{~kg}$ korrespondiert mit einem Blutdruckanstieg von 2 bis $3 \mathrm{mmHg}$ und mit einem Cholesterinanstieg um 10 bis $15 \mathrm{mg} / \mathrm{dl}$. 


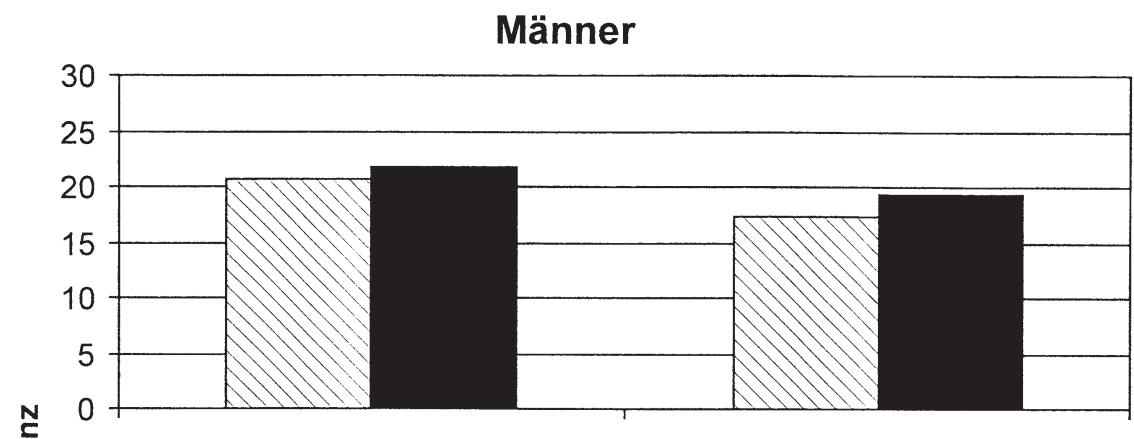

व

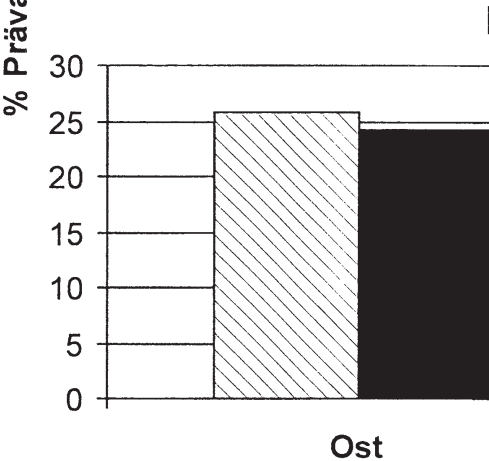

Frauen

1991

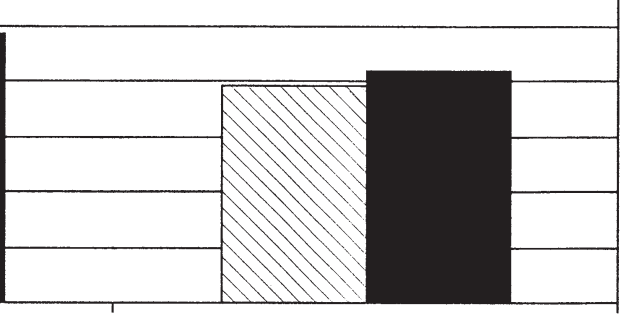

West

1998

Abb. $1 \Delta$ Prävalenz der Adipositas (Body Mass Index $\geq 30 \mathrm{~kg} / \mathrm{m}^{2}$ ) in Deutschland bei Männern und Frauen im Alter von 25 bis 69 Jahren. Die Ergebnisse der Gesundheitssurveys zeigen bei Frauen eine höhere Prävalenz als bei Männern, in den östlichen Bundesländern eine höhere Prävalenz als in den westlichen und von 1991 bis 1998 tendenziell eine Zunahme der Adipositas

Neben diesen univariaten Analysen gibt es multivariate Untersuchungen zur Bedeutung von Adipositas als „unabhängigem" kardiovaskulären Risikofaktor. In der Chicago Western Electric Studie, einer der klassischen epidemiologischen Kohortenstudien, ergab sich nach Berücksichtigung von Rauchen, Alkohol, EKG-Auffälligkeiten und Schulbildung eine signifikante Assoziation zwischen BMI und der koronaren Mortalität [11]. Bei zusätzlicher Adjustierung für systolischen Blutdruck und Cholesterin war die signifikante Assoziation nicht mehr zu sehen. Die Analysen in der Framingham-Studie ergaben andere Resultate [12]. Adipositas erwies sich als signifikanter unabhängiger Risikofaktor für koronare Herzkrankheit mit einem langfristigen relativen Risiko (RR) von 1,5, Herzinsuffizienz (RR 1,9), Schlaganfall (RR 2,0) und plötzlichen Herztod (RR 2,8). Bei der Berechnung des prädiktiven Wertes von Adipositas wurde für Patientenalter, Cholesterin, Blutdruck, Rauchen, linksventrikuläre Hypertrophie und Glukoseintoleranz adjustiert.
Der Zusammenhang zwischen BMI und Gesamtsterblichkeit wurde schon in den 8oer-Jahren in Norwegen untersucht [13]. Bei Frauen stieg die altersadjustierte Gesamtsterblichkeit ab einem BMI von 25 leicht und ab einem BMI von 30 deutlich an. Bei Männern ergab sich ein ähnliches Bild.

\section{"Die Lebenserwartung wird bereits bei Bestehen einer geringgradigen Adipositas reduziert."}

In einer groß angelegten prospektiven Kohortenstudie an mehr als 1 Mio Erwachsenen in den USA zeigte sich die geringste Gesamtsterblichkeit bei einem BMI von 23,5 bis 24,9 [14]. Gemessen an Personen mit diesem BMI, nahm die Gesamtsterblichkeit mit ansteigendem BMI kontinuierlich zu und war bei sehr hohem BMI um 2-2,5 erhöht. Für kardiovaskuläre Todesfälle war das relative Risiko sogar fast dreifach erhöht im Vergleich zur normalgewichtigen Gruppe (Abb.3). Auch im Bereich von BMI 20-25 scheint das kardiovaskuläre Risiko bereits zuzunehmen. Die Frage des „optimalen“ Gewichts unter kardiovaskulären Gesichtspunkten ist daher nicht einheitlich zu beantworten [15].

\section{Genetische Aspekte}

An der wichtigen Bedeutung genetischer Faktoren für die Veranlagung einer Adipositas besteht heute kein Zweifel mehr. So zeigen eine Vielzahl von Zwillingsuntersuchungen, dass die Variabilität des Körpergewichts in hohem Maße (60-80\%) vererbt wird [16]. Adipositas in der Kindheit ist ein deutlicher Prädiktor für Adipositas auch im Erwachsenenalter, besonders wenn ein Elternteil oder beide Eltern übergewichtig sind. Von den im Alter von drei bis fünf Jahren übergewichtigen Kindern entwickelten im Erwachsenenalter 24\% Adipositas, wenn die Eltern normalgewichtig waren, jedoch $62 \%$, wenn mindestens ein Elternteil übergewichtig war.

\section{"Genetische Aspekte spielen bei der Entwicklung von Adipositas eine wichtige Rolle."}

Die Bedeutung genetischer Faktoren für die Veranlagung zur Adipositas ist nicht überraschend, da die Fähigkeit, in Zeiten des Überflusses Energiereserven für Notzeiten anzuhäufen, zu den elementaren Eigenschaften lebender Organismen gehört. Unter den heute herrschenden Lebensbedingungen des Nahrungsüberangebots und Bewegungsmangels führt ein solches genetisches Programm für Notzeiten zwangsläufig zur Adipositas [17]. In der heutigen Wohlstandsgesellschaft bedeutet dies für diejenigen, bei denen das Programm an sich optimal funktioniert, einen lebenslangen Kampf „wider die eigene Natur“. Es verwundert daher kaum, dass nahezu alle bisherigen Ansätze, durch eine bewusste Verhaltensmodifikation das Körpergewicht zu kontrollieren, über kurz oder lang zum Scheitern verurteilt waren. Die hohe Rezidivrate und entmutigende Misserfolge nahezu aller konventionellen Interventionsprogramme zeigen, dass in der Praxis langfristige Gewichtsreduktionen nur in den seltensten Fällen zu erzielen waren [18]. Allerdings zeigen neue Therapieansätze, dass der Adipositas durchaus begegnet werden kann, sie also nicht 


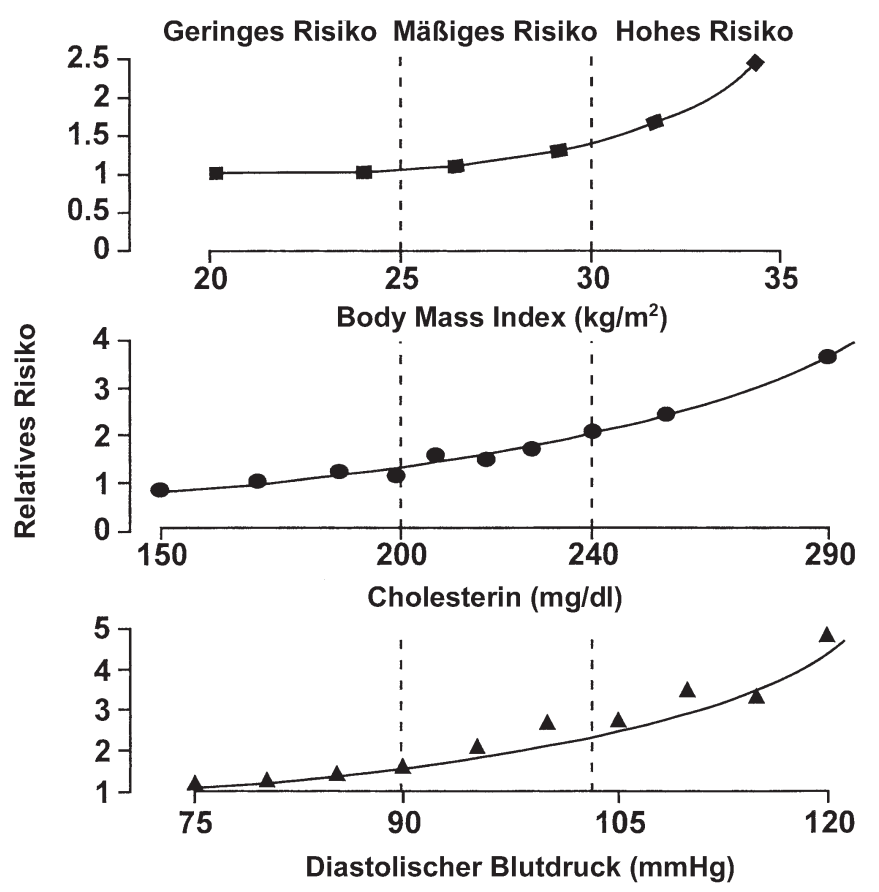

Abb.2 Das Risikopotential des Body Mass Index verläuft ähnlich wie bei Cholesterin und Blutdruck. Bei einer Zunahme wird das relative Risiko für Herz-Kreislauf-Erkrankungen erhöht [10]

als genetisch bedingtes Schicksal hingenommen werden muss (s. hierzu das Kapitel „Therapieansätze“ sowie den Beitrag von Pudel in diesem Heft).

In den letzten Jahren ist die Vielfalt und Komplexität der Mechanismen der Gewichtsregulation deutlich geworden. So wurden inzwischen eine Vielzahl von Faktoren entdeckt, wie das adipozytäre Hormon Leptin, die uncoupling Proteine, die $B_{3}$-adrenergen Rezeptoren, das agouti-related peptide, das Pro-Opiomelanocortin-System u. a., die in komplexer Weise Einfluss auf den Energiestoffwechsel aber auf das Nahrungsverhalten nehmen [19]. Mutationen in den einzelnen Komponenten dieser Systeme können sowohl beim Menschen als auch in Tiermodellen zur Entwicklung der Adipositas führen. Auch wenn solche Fälle wertvolle Hinweise für die biologische Funktion der Energieregulation liefern, so bleibt die Bedeutung genetischer Untersuchungen für die Diagnostik, Prognostik und Therapie der Adipositas vorerst abzuwarten.

\section{Kosten}

Für Folgen des Übergewichts werden in Deutschland jährlich ungefähr 10 Milliarden Euro ausgegeben [20]. Diese Berechnung umfasst direkte und indirekte
Kosten unter Berücksichtigung der wichtigsten Komorbiditäten bei Adipositas und beruht auf Daten der frühen 9oer-Jahre. Aus den USA liegen detaillierte Daten vor, die zeigen, dass die jährlichen direkten Krankheitskosten mit dem BMI deutlich ansteigen. Verursacht werden diese Kosten durch die mit Adipositas assoziierten Erkrankungen. Insbesondere beim Typ-2-Diabetes liegen die direkten Krankheitskosten ca. doppelt so hoch bei Patienten mit einem BMI von 25-29 und um ein Vielfaches höher bei Personen mit einem BMI >29, verglichen mit Normalgewichtigen [21]. Auch die indirekten Kosten, gemessen an Krankheitstagen und frühzeitigen Berentungskosten, liegen bei übergewichtigen Personen deutlich höher als bei Normalgewichtigen [22].

\section{Therapieansätze}

Wie bereits oben erwähnt, ist der langfristige Erfolg konventioneller Maßnahmen zur Gewichtsreduktion enttäuschend. Dennoch haben Interventionsversuche zur Gewichtsreduktion mittlerweile eine große Public-Health-Bedeutung erlangt. Der durchschnittliche Erwachsene unternimmt nach Studien in den USA alle zwei Jahre einen Versuch zur Gewichtsreduktion [23, 24]. Die am häufigsten angewandten Methoden sind dabei körperliche Aktivität (58\% Frauen und 44\% Männer), Diät (52\% Frauen und $45 \%$ Männern) und Medikation (14\% Frauen und 7\% Männer) [25]. Selbstverständlich ist eine Verhaltensmodifikation mit Änderungen des Ernährungsverhaltens und eine Steigerung der körperlichen Bewegung Grundlage jeder Adipositastherapie. Wesentliche psychologische Aspekte in der Adipositastherapie werden im vorliegenden Heft von Pudel dargestellt [26].

Darüber hinaus können nach den neuesten Untersuchungen medikamentöse Maßnahmen den langfristigen Erfolg der Behandlung unterstützen oder gar sichern. Obwohl bereits in früheren Jahren der Einsatz von zentralnervös wirksamen Appetithemmern zu einer signifikanten Gewichtsreduktion in rund $40 \%$ der Studien führte [27], erwiesen sich solche Substanzen aufgrund einer Vielzahl von Nebenwirkungen für die Dauerbehandlung als ungeeignet. Auch der Einsatz neuerer Substanzen wie Fenfluramin oder Dexfenfluramine ist trotz effektivem Gewichtsverlust [28, 29,30] wegen des Auftretens von pulmonaler Hypertension und Herzklappenfehlern [31] nicht zu vertreten. Serotonerge Substanzen erwiesen sich ebenfalls nur für begrenzte Zeit als geeignet [32]. Heute sind lediglich zwei Substanzen zur Behandlung der Adipositas in den USA und Europa zugelassen, nämlich der Noradrenalin- und Serotoninwiederaufnahmehemmer Sibutramin [33] und der Lipaseinhibitor Orlistat [34]. Für beide Substanzen liegen inzwischen randomisierte kontrollierte Studien mit einer Dauer von bis zu zwei Jahren vor. Trotz unterschiedlicher Begleiterscheinungen führt die 5 bis 10\%ige Gewichtsreduktion mit beiden Substanzen zu einer signifikanten Verbesserung vieler metabolischer und hämodynamischer (bei Orlistat) Parameter. Ob dies auch langfristig zu einer Senkung der kardiovaskulären Mortalität führt, bleibt abzuwarten.

Chirurgische Maßnahmen, wie z. B. das Gastric-Banding, haben sich ebenfalls, besonders bei Patienten mit einem BMI $>40 \mathrm{~kg} / \mathrm{m}^{2}$, bewährt [35]. Limitiert wird der Einsatz jedoch durch hohe Komplikationsraten, die z. T. auf die hohe perioperative Morbidität der adipösen Patienten zurückzuführen sind. 

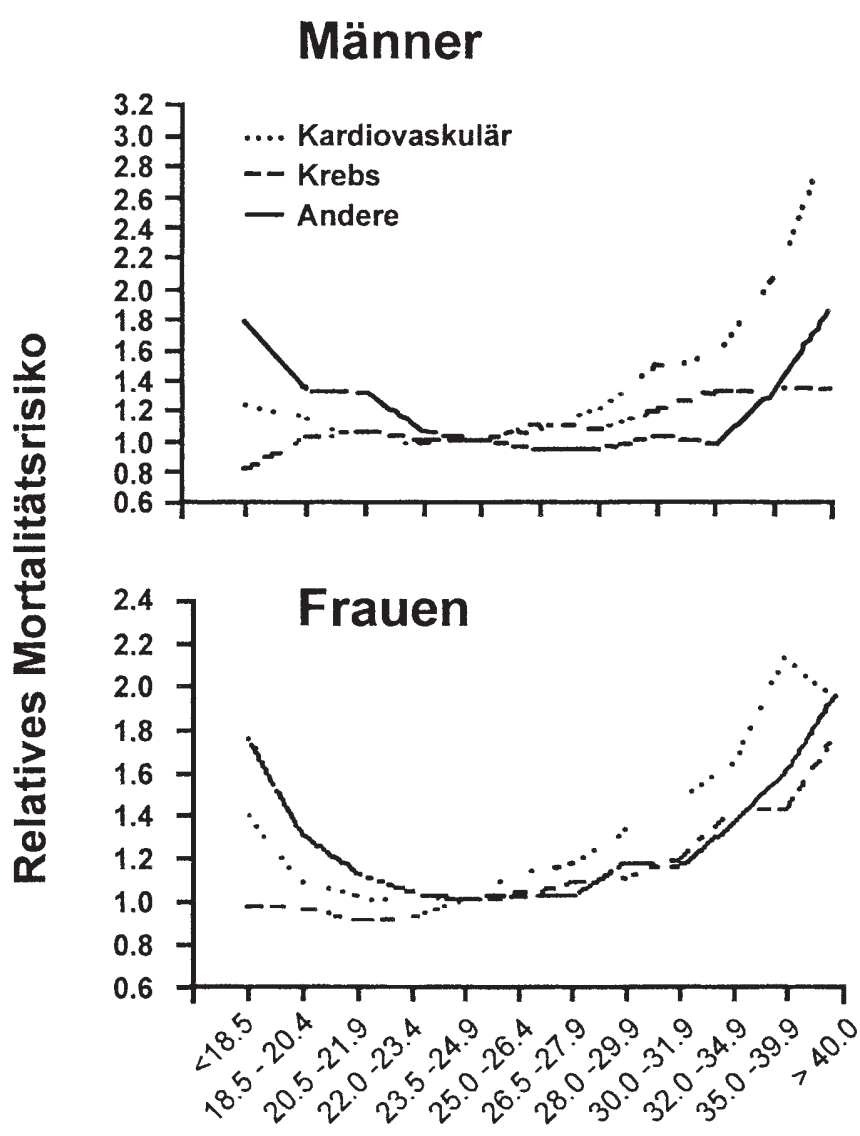

\section{Body Mass Index}

Abb. 3 Relatives Mortalitätsrisiko durch kardiovaskuläre Erkrankungen, Krebs und alle anderen Ursachen bei Männern und Frauen in Abhängigkeit vom Body Mass Index [14]. Die Referenzkategorien mit relativem Risiko 1 werden von den Personen mit einem Body Mass Index 23,5-24,9 kg/m² gebildet

In jedem Fall bedarf die erfolgreiche Behandlung der Adipositas langfristiger, in manchen Fällen lebenslanger Therapiekonzepte. Von kurzfristigen Ansätzen ist grundsätzlich abzuraten, da sie eher zu einer Verschlimmerung der Adipositas (Jojo-Effekt) führen können, und ausgeprägte Gewichtsfluktuationen mit einem erhöhten kardiovaskulären Risiko verbunden sind [36].

\section{Zusammenfassung und Ausblick}

Adipositas ist eine ernst zu nehmende Erkrankung, die bei global ansteigender Tendenz inzwischen über $20 \%$ der erwachsenen Bevölkerung in westlichen Industriegesellschaften betrifft (Frauen meist stärker als Männer). In Deutschland ist die Prävalenz in den östlichen Bundesländern bei Männern etwas und bei Frauen deutlich höher als in den westlichen. Adipositas ist mit verschiedenen gravierenden Gesundheitsproblemen assoziiert, insbesondere mit den kardiovaskulären Risikofaktoren. Darüber hinaus hat Adipositas ein unabhängiges Risikopotenzial für das Auftreten von Herz-Kreislauf-Erkrankungen.

Bisherige Interventionsstudien haben unterschiedliche und häufig enttäuschende Ergebnisse erbracht. Die Endpunkte dieser Studie beziehen sich bisher vor allem auf Gewichtsreduktion oder auf Surrogatendpunkte wie kardiovaskuläre Risikofaktoren. Weitere Langzeituntersuchungen mit klinischen Ereignissen und Mortalität als Endpunkten sind erforderlich, um den möglichen Nutzen von konsequenter Gewichtsreduktion einzuschätzen.

Nach wie vor gilt das Zitat von Stunkard: „Most obese persons will not stay in treatment, most will not lose weight, and of those who lose weight, most will regain it" [36]. Daher müssen kontinuierliche integrierte Konzepte für Therapie und Prävention entwickelt werden in Kombination von Diät, körperlicher Aktivität und pharmakologischer Therapie (ggf. sogar zusätzlicher chirurgischer Intervention). Adipositas muss vom Arzt und vom Betroffenen selbst als ernst zu nehmende Krankheit akzeptiert werden und damit nicht nur als ästhetisches Problem oder Ausdruck von „Zügellosigkeit“ bzw. „Willensschwäche“ verharmlost werden.

\section{Literatur}

1. Shakespeare W (1976) Julius Caesar. Universal-Bibliothek 9816, Reclam, Stuttgart

2. von Dusch TK (1868) Lehrbuch der Herzkrankheiten. Engelmann, Leipzig

3. Pi-Sunyer FX (1993) Medical hazards of obesity. Ann Intern Med 119:655-660

4. Wechsler JG, Schusdziarra V, Hauner H, Gries FA (1996) Therapie der Adipositas. Dtsch Ärztebl 93:A-2214-2218

5. Kuczmarski RJ, Flegal KM, Campbell SM, Johnson CL (1994) Increasing prevalence of overweight among U.S. adults. The National Health and Nutrition Examination Surveys, 1960 to 1991. JAMA 272:205-211

6. WHO MONICA Project (1989) Risk Factors. Int J Epidemiol 18 (3) [Suppl 1]:46-55

7. Thefeld W (2000) Verbreitung der Herz-Kreislauf-Risikofaktoren Hypercholesterinämie, Übergewicht, Hypertonie und Rauchen in der Bevölkerung. Bundesgesundheitsbl Gesundheitsforsch Gesundheitsschutz 43:415-423

8. EUROASPIRE Study Group (1997) A European society of cardiology survey of secondary prevention of coronary heart disease. Principal Results Eur Heart J 18:1569-1582

9. Willich SN, Müller-Nordhorn J, Kulig M et al. (2001) Cardiac risk factors, medication, and recurrent clinical events after acute coronary disease. Eur Heart J 22:307-313

10. Bray GA (1998) Obesity: a time bomb to be defused.Lancet 352:160-161

11. Spataro JA, Dyer AR, Stamler J, Shekelle RB (1994) Measure of adiposity and CHD mortality in the Chicago Western Electric Study. Abstract presented at 34th Annual Conference on Cardiovascular Disease Epidemiology and Prevention, Tampa, Florida, March 1994

12. Hubert HB, Feinleib M, McNamara PM, Castelli WP (1983) Obesity as an independent risk factor for cardiovascular disease: a 26-year follow-up of participants in the Framingham Heart Study. Circulation 67:968-977

13. Waaler $\mathrm{H}$ (1984) Height, weight and mortality. The Norwegian experience. Acta Med Scand Suppl 679:1-56 
14. Calle EE, Thun MJ, Petrelli JM, Rodriguez C, Heath CW (1999) Body mass index and mortality in a prospective cohort of U.S. adults. N Engl J Med 341:1097-1105

15. Liu S, Manson JE (2001) What is the optimal weight for cardiovascular health? BMJ 322:631-632

16. Stunkard AJ (1996) Current views on obesity. Am J Med 100:230-236

17. Sharma AM, Deprés JP (2001) Obesity: the dire consequence of a genetic program for survival? J Mol Med 79:6-7

18. Kramer FM, Jeffrey RW, Forster JL, Snell MK (1989) Long-term follow up of behavioural treatment for obesity: patterns of weight regain among men and women. Int J Obes 13:123-136

19. Hamann A, Münzberg H, Algenstedt P, Tafel J (im Druck) Molekulare Grundlage der Adipositas. Herz 26

20. Schneider R (1996) Relevanz und Kosten der Adipositas in Deutschland. Ernährung Umschau 43:369-374

21. Narbro K, Jonsson $E$, Larsson B, Waaler $H$, Wedel H, Sjöstrom L (1996) Economic consequences of sick-leave and early retirement in obese Swedish women. Int J Obes Relat Metab Disord 20:895-903

22. Wolf AM, Colditz GA (1996) Social and economic effects of body weight in the United States. Am J Clin Nutr 63 (3) [Suppl]:466-469

23. Levy AS, Heaton AW (1993) Weight control practices of U.S. adults trying to lose weight. Ann Intern Med 119:661-666

24. Serdula MK, Collins ME, Williamsen DF et al. (1993) Weight control practices of U.S. adolescents and adults. Ann Intern Med 119:667-671

25. Wadden TA (1993) Treatment of obesity by moderate and severe caloric restriction. Results of clinical trials. Ann Intern Med 119:688-693

26. Pudel V (2001) Psychologische Therapieansätze in der Adipositastherapie. Bundesgesundheitsbl Gesundheitsforsch Gesundheitsschutz 44 (in press)

27. Bray GA (1993) Use and abuse of appetitesuppressant drugs in the treatment of obesity. Ann Intern Med 119:749-757

28. Guy-Grand B, Apfelbaum M, Crepaldi G, Gries A Lefebvre $P$, Turner $P$ (1989) International trial of long-term dexfenfluramine in obesity. Lancet 2:1142-1145

29. Finer N (1992) Body weight evolution during dexfenfluramine treatment after initial weight control. Int J Obes Relat Metab Disord 16 (3) [Suppl]:5-14
30. Breum L, Pederson JK, Alhstrom F, Frimodt-Moller J (1994) Comparison of ephedrine/caffeine combination and dexfenfluramine in the treatment of obesity: a double blind multicenter trial in general practice. Int J Obes Relat Metab Disord 18 (2):99-103

31. Editorial (1997) Diet pills Redux. N Engl J Med 337:629-630

32. Gray DS, Fujioka K, Devine W, Bray GA (1992) Fluoxetine treatment of the obese diabetic. Int J Obes Relat Metab Disord 16 (3) [Suppl]:193-198

33. James WP, Astrup A, Finer $\mathrm{N}$ et al. (2000) Effect of sibutramine on weight maintenance after weight loss: a randomised trial. STORM Study Group. Sibutramine Trial of Obesity Reduction and Maintenance. Lancet 356:2119-2125

34. Sjöström L, Rissanen A, Andersen T et al. (1998) Randomised placebo-controlled trial of orlistat for weight loss and prevention of weight regain in obese patients. Lancet 352:167-172

35. Sjöström L (2000) Surgical intervention as a strategy for treatment of obesity. Endocrine 13:213-230

36. Higgins M, D'Agostino R, Kannel W, Cobb J (1993) Benefits and adverse effects of weight loss. Observations from the Framingham Study. Ann Intern Med 119:758-763

37. Stunkard AJ (1958) The management of obesity. NY State J Med 58:79-87

\section{E. Deutsch, A.W. Bender, R. Eckstein, R. Zimmermann Transfusionsrecht}

Wissenschaftliche Verlagsgesellschaft $\mathrm{mbH}$, Stuttgart, 2001, 416 S., kartoniert, DM 96,(ISBN - 3-8047-1814-0)

Das kürzlich erschienene Buch „Transfusionsrecht" versteht sich nicht als Kommentar im klassischen Sinn, sondern als eine problemorientierte Betrachtung, die das Umfeld der einzelnen Bestimmungen des Transfusionsgesetzes beleuchtet. Diesem Anspruch wird das 416 Seiten starke, mit zahlreichen interessanten Informationen und Überlegungen angefüllte Werk durchaus gerecht.

Das Werk gliedert sich in mehrere Abschnitte und Anhänge; die Gliederung ist sehr detailliert und klar. Die normalerweise wohl eher dem Juristen geläufige Verwendung von durchlaufenden Randnummern macht die zahlreichen und angesichts der Komplexität der Materie auch erforderlichen Querverweise auch für den Mediziner übersichtlicher und ist sehr gut zu handhaben. Die Einführung in die Thematik in dem ersten Abschnitt mit der Überschrift "Grundlagen" beginnt mit einem historischen Überblick zur Entwicklung der Transfusionsmedizin, gefolgt von der speziellen Vorgeschichte des Transfusionsgesetzes und erscheint sehr gelungen. In den weiteren Abschnitten werden als weitere Schwerpunkte detailliert die Vorschriften hinsichtlich der Spende, der Anwendung von Blutprodukten, der Rückverfolgung und des Meldewesens abgehandelt. In den Anhängen werden Informationen zu Dokumentationspflichten, zum Qualitätsmanagement und schließlich der Text des Transfusionsgesetzes angeboten.

Das Buch verbindet viele Fachinformationen mit sehr eingehenden juristischen Erläuterungen und Interpretationen. Zum Teil wird auch ein Disput über die vom Gesetzgeber gewählten gesetzestechnischen Lösungswege eröffnet, der die Diskussion in den einschlägigen juristischen Fachkreisen stimulieren dürfte. Insgesamt handelt es sich um ein Werk, dass vermutlich weniger zum einfachen Durchlesen angetan ist, sondern eher ein Kompendium darstellt, dass mit seiner gründlichen Darstellungsweise und vielen Literaturhinweisen an zahlreichen Stellen zu einer vertieften medizinisch-fachlichen und juristischen Auseinandersetzung mit der wichtigen Materie des Transfusionswesens anregt und anleitet.

Es kann daher erwartet werden, dass die mit dem Transfusionsgesetz konfrontierten Ärzte und Juristen aus der Lektüre dieses interdisziplinären Buches Gewinn ziehen werden. Es handelt sich um eine sehr detaillierte und fundierte Arbeit, die sicher einen entsprechenden Stellenwert unter der Literatur zum Transfusionsrecht einnehmen wird. 